\title{
Electrical Parameters of Low-Voltage Power Distribution Cables Used for Power-Line Communications
}

\author{
Georgios T. Andreou, Member, IEEE, and Dimitris P. Labridis, Senior Member, IEEE
}

\begin{abstract}
Many models proposed in the literature to describe low-voltage power distribution networks in consumer premises as communication media require knowledge of the electrical parameters of the cables comprising these networks. These parameters are nevertheless affected by a large number of factors which may vary greatly from case to case, making it thus very difficult to achieve an exact estimation about them. In this work, a study of the electrical parameters of two cable types widely used in residential lowvoltage power distribution networks is presented. Moreover, a finite-element approach is used for the verification of the results of the theoretical model concerning the series impedance per unit length of the cable type under study with respect to its normal operational conditions.
\end{abstract}

Index Terms-Cable impedance, finite-element method (FEM), power-line communications.

\section{INTRODUCTION}

$\mathbf{R}$ ELIABLE high-rate data transmission via the low-voltage (LV) power distribution network has become a reality over the past years, with more commercial applications appearing as time passes. There are nevertheless yet questions to be answered about the analytical determination of the medium's properties.

The proper modeling of LV power distribution networks in consumer premises as communication media is a matter that may provide a boost in the data transmission rates achieved as yet, improving thus the chances of power-line communications as a commercial solution against opposing technologies. Many of the models proposed in the literature attempt to describe the cables which comprise typical LV power distribution networks by using their lumped or distributed electrical parameters (i.e., their resistance $R^{\prime}$, inductance $L^{\prime}$, conductance $G^{\prime}$, and capacitance $C^{\prime}$ per unit length [1]-[3]). An exact theoretical estimation of these parameters is nevertheless very difficult for most LV networks since the large number of factors that affect them, which cannot be accounted for in all cases. In some cases, the analytical determination of some of the above parameters is even practically impossible.

In the first part of this work, a thorough study concerning the theoretical calculation of the electrical parameters of LV cable based on existing literature is presented. The frequency dependency of the parameters under study is taken into account whenever possible.

Manuscript received March 7, 2006. Paper no. TPWRD-00121-2006.

The authors are with the Department of Electrical and Computer Engineering, Aristotle University of Thessaloniki, Thessaloniki 54124, Greece (e-mail: gandreou@auth.gr; labridis@auth.gr).

Digital Object Identifier 10.1109/TPWRD.2006.881577

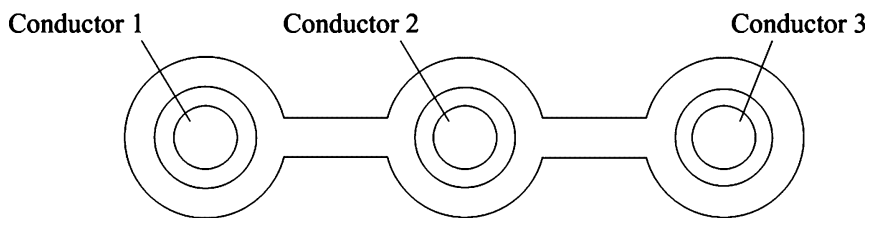

Fig. 1. Typical conductor arrangement for an NYIF cable.

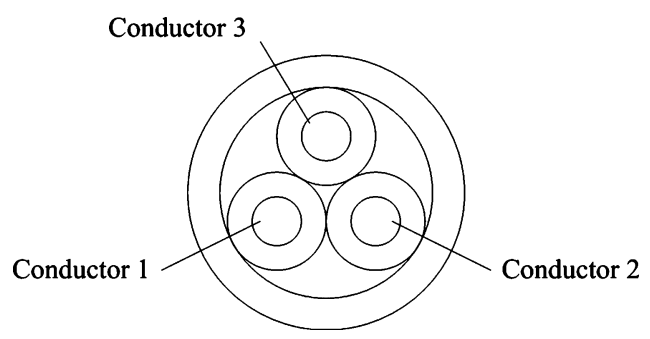

Fig. 2. Typical conductor arrangement for an NYM cable.

In the second part, a finite-element approach is used to derive operational series impedances of two cable types widely used in residential power installations with respect to its normal operational conditions. This approach is based on a finite-element method (FEM) formulation of the electromagnetic diffusion equation. The cables' frequency dependent series impedances are calculated in this case taking into account the geometric configuration, skin and proximity effect, and eddy currents flowing in other possibly existing conducting paths.

The finite-element approach is used in the frequency range up to $100 \mathrm{MHz}$, and the contribution of the presence of the earth to the cable's electrical parameters is also examined. The inability of the existing theory to account for all phenomena in the frequency range under study leads to expected differences in the FEM results as compared to theoretical calculations, which are denoted and explained.

\section{CABle Selection}

The cable types used for residential power distribution networks in Greece are mainly three: single-conductor, single-core cables (NYA per VDE 0250), and flat three-conductor, singlecore cables (NYIF per VDE 0250, Fig. 1), and circular threeconductor cables, both single- and multicore (NYM per VDE 0250, Fig. 2).

The conductors of all these cable types are typically made from copper, while special PVC compounds are used for their 
insulation. In residential installations, two conductors are used in any given circuit for power distribution (phase and return conductor, respectively), and a third conductor is used as protective earthing where necessary.

The two latter cable types are modeled in this work, for a typical conductor cross section of $2.5 \mathrm{~mm}^{2}$.

\section{Theoretical CAlculation of the ELECTRICAL PARAMETERS}

\section{A. Resistance Per Unit Length}

The resistance per unit length $R^{\prime}$ is mainly affected by the material and cross-sectional area of the conductors comprising the cable, its geometry (due to the proximity effect), the current frequency (due to the skin effect), the temperature, and the presence of the earth. Analytical expressions exist over the past decades, which may account for all of the above factors.

The dc resistance of a conductor at a temperature of $20{ }^{\circ} \mathrm{C}$ can be calculated with

$$
R_{\mathrm{dc} 20}=\frac{\rho \cdot l}{A}
$$

where $\rho$ is the special resistivity of the conductor $(\rho=1.7241$. $10^{-2} \Omega \cdot \mathrm{mm}^{2} / \mathrm{m}$ for copper and $\rho=2.8264 \cdot 10^{-2} \Omega \cdot \mathrm{mm}^{2} / \mathrm{m}$ for aluminium conductors [4]), $l$ is the length of the conductor, and $A$ is its cross-sectional area.

Given the above resistance for any conductor, we can calculate its dc resistance at any temperature with

$$
R_{\mathrm{dc}}=R_{\mathrm{dc} 20}\left[1+\alpha_{20}\left(\theta_{L}-20^{\circ} \mathrm{C}\right)\right]
$$

where $\alpha_{20}$ a temperature coefficient for the electrical resistance at $20^{\circ} \mathrm{C}\left(\alpha_{20}=3.93 \cdot 10^{-3}\right.$ for copper and $\alpha_{20}=4.03 \cdot 10^{-3}$ for aluminium conductors [4]).

The frequency-dependent increase of the resistance due to the skin effect can be calculated with the help of Bessel functions as an ac-dc resistance ratio [5]

$$
\frac{R_{\mathrm{ac}}}{R_{\mathrm{dc}}}=\frac{u / 2 \cdot B}{A R}
$$

where $u$ is a factor related to the skin depth $\delta, A R$ is the area ratio factor (which is equal to 1 in the case of a solid conductor) and $B$ is related to the Bessel functions (ber, bei) as follows:

$$
\begin{aligned}
u & =r \cdot \frac{\sqrt{2}}{\delta} \\
\delta & =\frac{1}{\sqrt{\pi f \sigma \mu}} \\
B & =\frac{\operatorname{ber}(u) \cdot \operatorname{bei}^{\prime}(u)-\operatorname{bei}(u) \cdot \operatorname{ber}^{\prime}(u)}{\left(\operatorname{ber}^{\prime}(u)\right)^{2}+\left(\operatorname{bei}^{\prime}(u)\right)^{2}}
\end{aligned}
$$

where $r$ is the conductor radius, $f$ the current frequency, $\sigma$ the conductor special conductivity, and $\mu$ its magnetic permeability.

Nevertheless, these expressions apply only in the case of a continuous cable, as any discontinuity (such as a junction or an insulation fault) may lead to problems that are difficult to overcome. Such discontinuities will appear very often in practice due to imperfections during construction and the aging of residential power distribution networks.

The above study concerns the resistance per unit length of a single conductor. When calculating the circuit resistance per unit length of a single-phase transmission line, one simply has to double the corresponding resistance per unit length of one conductor. Some error is to be expected if the conductors are close to each other (as is the case for the cable types under study in this paper) due to the proximity effect.

The same holds true for the third conductor being present in the cable types under study here. This conductor can influence the overall resistance of the circuit formed by the other two conductors due to the proximity effect, which is ignored in (3).

\section{B. Inductance Per Unit Length}

The inductance per unit length $L^{\prime}$ of a single-phase transmission line can be calculated as the sum of two partial inductances, the internal inductance per unit length $L_{i}^{\prime}$, which is the result of the magnetic field energy inside the conductors, and the external inductance per unit length $L_{o}^{\prime}$, which stems from the magnetic-field energy outside the conductors [6]. At very low frequencies, for which the current is uniform over the cross-sectional area of the conductors, the internal inductance per-unit length of the single-phase transmission line (usually denoted as $L_{\mathrm{dc}}^{\prime}$ in this case) can be calculated with (7) as two times the respective internal inductance of one conductor

$$
L_{\mathrm{dc}}^{\prime}=\frac{\mu}{4 \pi}
$$

This internal inductance is independent of all geometrical parameters, provided that the wire is round. However, as the current frequency rises, the current distribution will not be uniform over the cross section of the conductors due to the skin effect. This phenomenon can be accounted for, and the corresponding reduction in the circuit internal inductance per unit length can be calculated as an ac-dc ratio with (8) [5]

$$
\frac{L_{i}^{\prime}}{L_{\mathrm{dc}}^{\prime}}=\frac{4}{\sqrt{2}} \frac{\delta}{r}\left[\frac{\operatorname{ber}(u) \cdot \operatorname{ber}^{\prime}(u)-\operatorname{bei}(u) \cdot \operatorname{bei}^{\prime}(u)}{\left(\operatorname{ber}^{\prime}(u)\right)^{2}+\left(\operatorname{bei}^{\prime}(u)\right)^{2}}\right] .
$$

The external circuit inductance per unit length of a singlephase transmission line is independent from current frequency and can be calculated with (9) [5]

$$
L_{o}^{\prime}=\frac{\mu}{\pi} \ln \left[\frac{S+\sqrt{S^{2}-4 r^{2}}}{2 r}\right]
$$

where $S$ is the distance between the centers of the two conductors and $r$ is the radius of the conductors.

Note that this external circuit inductance can be calculated with the help of the reciprocity theorem [5], as long as TEM approximations can be made for the transmission line under study. In this case, the external circuit inductance can be associated with the respective capacitance with (10)

$$
L_{o}^{\prime}=\frac{\mu \varepsilon}{C^{\prime}} .
$$


Concerning the third conductor of the cable types under study in this paper and its contribution to the overall inductance per unit length of the circuit formed by the other two conductors, the same is valid as for the circuit resistance per unit length. The cables' third conductor is typically grounded in residential LV installations; therefore, it may influence the circuit inductance only due to the proximity effect.

However, the external inductance of a cable will generally alter in the presence of current carrying conductors running in parallel with the cable, as the conductor geometry will thus change. This introduces an additional factor of uncertainty in our calculations, as LV power distribution networks inside consumer premises usually include cables from different circuits which run along the same routes.

\section{Capacitance Per Unit Length}

The capacitance per unit length $C^{\prime}$ of a cable can also be divided in two partial capacitances, namely the capacitance between the conductors and the capacitance between any of the conductors and the earth.

The capacitance per unit length between two conductors $C_{c}^{\prime}$ can be calculated with (11) [5]

$$
C_{c}^{\prime}=\frac{\pi \varepsilon_{0} \varepsilon_{r}}{\ln \left[\frac{S+\sqrt{S^{2}-4 r^{2}}}{2 r}\right]}
$$

where $\varepsilon_{r}$ is the permittivity of the dielectric separating the two conductors, $S$ is the distance between the centers of the two conductors, and $r$ is the radius of the conductors.

An important issue is that of the dielectric permittivity $\varepsilon_{r}$, which is generally frequency dependent, a fact often overlooked in literature. For typical polyvinyl chloride (PVC) compounds used for LV power cables [7], this is a matter of great importance, as the values of the permittivity range from 6-7 to 3 for frequencies up to $100 \mathrm{MHz}$ [8].

The real problem with the calculation of the capacitance, however, is that of the determination of the cable's capacitance to earth. In the case of residential LV power distribution cables, the absence of a metallic cover over the conductors makes the analytical calculation of this capacitance impossible as, in this case, the extent of the electrostatic field is indeterminable [4]. Moreover, inside a building, the very definition of the properties of an equivalent ground, which may be used for the calculation of a cable capacitance, is a daring task, complicated even more by the presence of a considerable amount of other electric-field sources.

With the absence of the cable's partial capacitance to earth, the error factor will be, in many cases, insignificant due to the cable's small dimensions, as compared to its distance from the ground. Yet, there may be several situations where the error will be substantial, especially if the cable under study is located in the vicinity of other electric-field sources.

In the case of the capacitance per unit length, we may no longer neglect the influence of the third conductor present in the cable types under study. Considering the arbitrary conductor geometry shown in Fig. 3, which corresponds to an NYM cable,

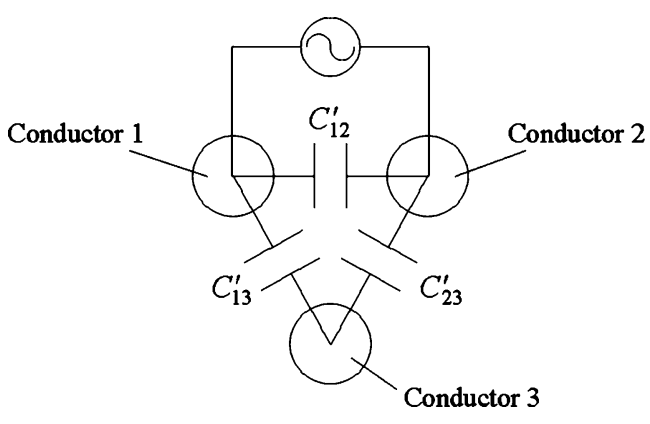

Fig. 3. Equivalent capacitances in an NYM-cable conductor geometry.

the capacitance per unit length for the circuit formed by conductors 1 and 2 can be calculated with (12)

$$
C^{\prime}=C_{12}^{\prime}+\frac{C_{13}^{\prime} \cdot C_{23}^{\prime}}{C_{13}^{\prime}+C_{23}^{\prime}}
$$

The partial capacitances in (12) can be calculated with (11).

\section{Conductance Per Unit Length}

The problems concerning the evaluation of a cable's capacitance also affect the accurate estimation of its conductance per unit length $G^{\prime}$, as its value is usually calculated as the product of the cable's capacitive susceptance per unit length and the loss tangent of the dielectric material (13)

$$
G^{\prime}=\omega C^{\prime} \tan \delta
$$

Moreover, there is a significant contradiction in the literature about the values of the loss tangent used for PVC, probably due to the existence of various different PVC compounds. A value often (albeit mistakenly) used is that of $\tan \delta=0.01$ which, according to Paul [9], is an unrealistically large value in the frequency range up to $100 \mathrm{MHz}$.

Other authors seem to disagree in this point. Naidu and Kamaraju [10] propose a value of 0.015-0.02 for $\tan \delta$, whereas Heinhold [4] suggests, respectively, a value of 0.1 , and Tsuzuki et al. [11] proposes values of $\tan \delta$ that vary from 0.3 to 0.1 in the frequency range up to $30 \mathrm{MHz}$, based on their own measurements.

Again, the most accurate work concerning the specific PVC compounds used for residential LV power distribution cables may be that of von Hippel [8], who offers tabulated values of $\tan \delta$ that range from 0.1 to 0.03 for frequencies up to $100 \mathrm{MHz}$.

To understand the impact of the variation of the loss tangent's values, we will compare results for two different loss tangent values, namely $\tan \delta_{1}=0.01$ and $\tan \delta_{2}=0.1$ (a $900 \%$ rise). For any given value of the cable capacitance, (13) shows that there will be a corresponding $900 \%$ rise in the value of the cable conductance. However, (13) also shows that the cable's capacitive susceptance will be for the considered loss tangent values at least by one order greater than the cable's conductance. This leads to the result that the corresponding rise in the value of the cable's shunt admittance will only be $0.45 \%$. This explains the general lack of interest for the exact values of the loss tangent, as its contribution to the value of a cable's shunt admittance is practically negligible. 


\section{Finite-Element Method}

\section{A. General Formulation}

A system of $N$ parallel conductors, carrying root mean square (rms) currents $I_{i}(i=1,2, \ldots, N)$ is considered. The nonuniform current distribution inside the conductors influences the effective impedance of the conductors at a given frequency. The following matrix equation links voltages and currents in any conductor of the cable:

$$
\frac{\partial}{\partial z} \mathbf{V}=-\mathbf{Z}(\omega) \mathbf{I}
$$

where $\mathbf{V}$ is the voltage vector with respect to a reference, and $\mathbf{I}$ is the current vector. The elements of matrix $\mathbf{Z}(\omega)$ are the frequency-dependent series impedances per unit length, depending on the geometric configuration, skin and proximity effect, and eddy currents flowing in other conducting paths.

The problem itself could be greatly simplified, assuming that the per unit length complex voltage drop $\bar{V}_{i}$ on every conductor is known for specific current excitation. The mutual complex impedance $\bar{Z}_{i j}$ between conductor $i$ and another conductor $j$ carrying current $\bar{I}_{j}$, where all other conductors are forced to carry zero currents, is then given by

$$
\bar{Z}_{i j}=\frac{\bar{V}_{i}}{\bar{I}_{j}} \quad(i, j=1,2, \ldots, N) .
$$

The self impedance of a conductor may also be calculated from (14), by setting $\mathrm{i}=\mathrm{j}$. In such a case, the following procedure may be used for the calculation of the cable impedance matrix $\mathbf{Z}(\omega)$.

- A sinusoidal current excitation of arbitrary magnitude is applied sequentially to each conductor, while the remaining conductors are forced to carry zero currents. The corresponding voltages are recorded.

- Using (14), the $j$ th column of $\mathbf{Z}(\omega)$ may be calculated. This procedure is repeated $N$ times, in order to calculate the $N$ columns of $\mathbf{Z}(\omega)$.

The problem is then reduced to that of calculating the actual per unit length voltage drops, when a current excitation is applied to the conductors. This may be achieved by an FEM formulation of the electromagnetic diffusion equation.

The previously described cable, consisting of $N$ parallel conductors, is assumed to be long enough to ignore end effects. Furthermore, if the current density vector is supposed to be in the $z$ direction, the problem becomes two-dimensional, confined in the $x-y$ plane, in which the conductors' cross sections lie. The linear electromagnetic diffusion equation is described by the following system of equations [12]:

$$
\begin{aligned}
& \frac{1}{\mu_{0} \mu_{r}}\left[\frac{\partial^{2} \bar{A}_{z}}{\partial x^{2}}+\frac{\partial^{2} \bar{A}_{z}}{\partial y^{2}}\right]-j \omega \sigma \bar{A}_{z}+\bar{J}_{s z}=0 \\
& -j \omega \sigma \bar{A}_{z}+\bar{J}_{s z}=\bar{J}_{z} \\
& \iint_{S_{i}} \bar{J}_{z} d S=\bar{I}_{i}, \quad i=1,2, \ldots, N
\end{aligned}
$$

where $\bar{A}_{z}$ is the $z$ direction component of the magnetic vector potential (MVP).
In (17), the total current density $\bar{J}$ is decomposed in two components

$$
\bar{J}_{z}=\bar{J}_{e z}+\bar{J}_{s z}
$$

where $\bar{J}_{e z}$ is the eddy current density and $\bar{J}_{s z}$ the source current density, given by (20) and (21), respectively

$$
\begin{aligned}
& \bar{J}_{e z}=-j \omega \sigma \bar{A}_{z} \\
& \bar{J}_{s z}=-\sigma \nabla \Phi .
\end{aligned}
$$

FEM is applied for the solution of (16) and (17) with the boundary conditions of (18). Values for $\bar{J}_{s z_{i}}$ on each conductor $i$ of conductivity $\sigma_{i}$ are then obtained and (15) takes the form [13]

$$
\bar{Z}_{i j}=\frac{\bar{V}_{i}}{\bar{I}_{j}}=\frac{\bar{J}_{s z_{i}} / \sigma_{i}}{\bar{I}_{j}} \quad(i, j=1,2, \ldots, N)
$$

linking properly electromagnetic-field variables and equivalent circuit parameters. Finally, positive-, negative-, and zero-sequence impedances may be easily obtained. The positive-sequence series impedance matrix leads to the calculation of the operational cable resistance $R^{\prime}$ and inductance $L^{\prime}$ per unit length [13].

\section{B. Simulation Procedure}

In this work, we used an application software developed at the Power Systems Laboratory of the Department of Electrical and Computer Engineering in the Aristotle University of Thessaloniki, based on the aforementioned procedure. The input data of the application describe the problem geometry (taking into account the various material properties) and the initial current excitation of the conductors. Any given conductor may be considered to be energized, grounded, or open circuited.

At first, the FEM application has been used to calculate the electrical parameters of a single-phase circuit using the geometry of an NYM $3 \times 2.5 \mathrm{~mm}^{2}$ cable and disregarding the third conductor. Subsequently, the third conductor has also been used in order to demonstrate the differences in the circuit's series impedance due to its existence. In both cases, we also show the contribution of the earth to the circuit series impedance.

A similar procedure was also used for an NYIF $3 \times 2.5 \mathrm{~mm}^{2}$ cable geometry. Two different configurations were used in this case. In the first one, the circuit consisted of conductors 1 and 2 of Fig. 1, whereas in the second one, the circuit consisted of conductors 1 and 3 .

All calculations were performed in the frequency range up to $100 \mathrm{MHz}$. There is an inherent error factor due to truncation error, insignificant in most cases, as the finite-element method is an arithmetical method, whose precision is based on continuous iterations of ever-growing computational needs. In order to reduce this error, a local error estimator, based on the discontinuity of the instantaneous tangential components of the magnetic field, has been chosen as in [15] for an iteratively adaptive mesh generation in all examined cases.

\section{Two Conductor Circuit}

At first, the case of a single-phase circuit is examined using the geometry of an NYM $3 \times 2.5 \mathrm{~mm}^{2}$ cable and disregarding 


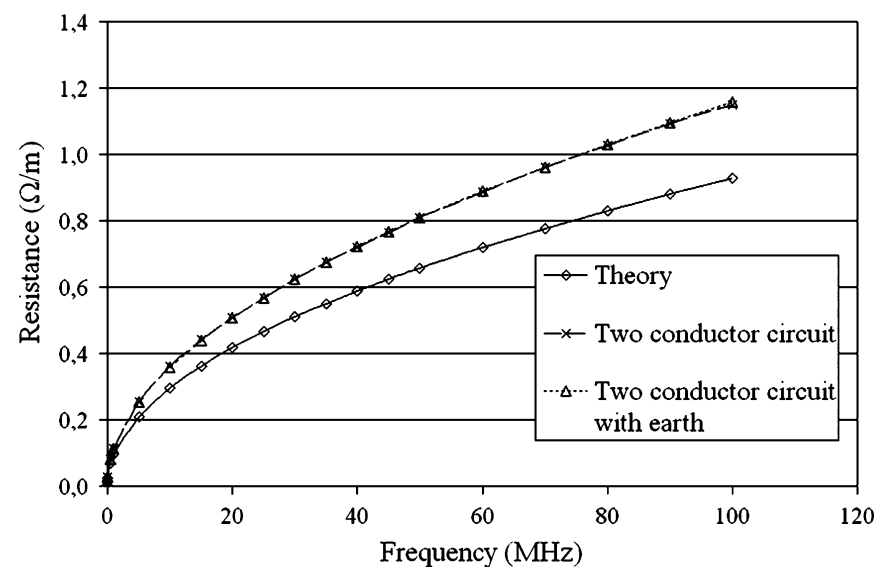

Fig. 4. Circuit resistance per unit length for an NYM $3 \times 2.5 \mathrm{~mm}^{2}$ cable, disregarding the third conductor.

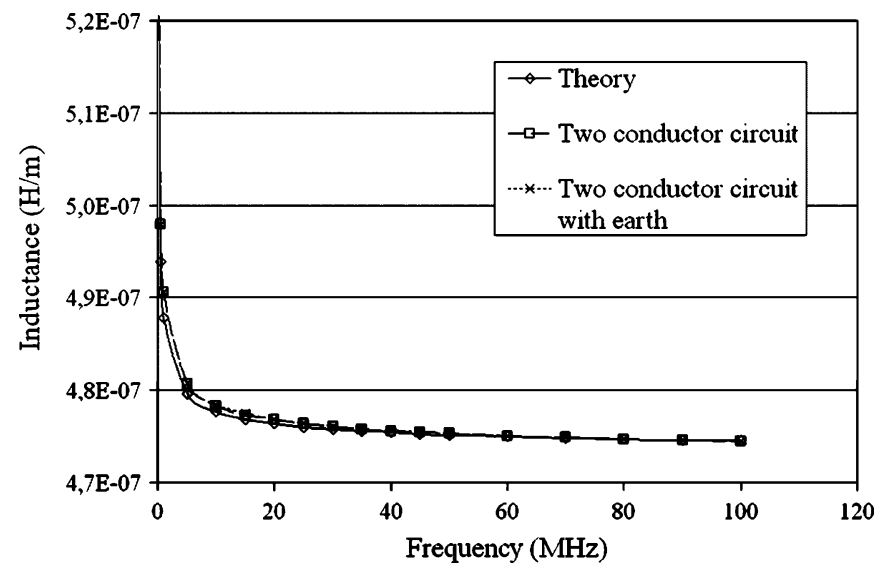

Fig. 5. Circuit inductance per unit length for an NYM $3 \times 2.5 \mathrm{~mm}^{2}$ cable, disregarding the third conductor.

the third conductor. Figs. 4 and 5 show, respectively, the circuit's resistance and inductance per unit length versus current frequency. The three curves in both figures represent, respectively, the theoretical calculations and FEM results for two different configurations, namely a circuit configuration without considering the presence of the earth, and a circuit configuration, where the conductors lie $0.5 \mathrm{~m}$ above ground (with an earth resistivity of $100 \Omega \cdot \mathrm{m})$.

In Fig. 4, an expected deviation between theoretical calculations and FEM results can be observed because of the proximity effect, which is not taken into account in the theoretical analysis. This deviation is $0.10 \%$ at base frequency, where the proximity effect is insignificant, and reaches up to $26.80 \%$ at $25 \mathrm{MHz}$.

In the case of the circuit inductance (Fig. 5), the proximity effect only affects the conductors' internal inductance, which, for this cable configuration, is significantly smaller than the external inductance. At low frequencies, the deviation reaches $6 \%$ (at base frequency), but its value remains below $0.5 \%$ for frequencies above $1 \mathrm{MHz}$.

The importance of the proximity effect becomes apparent if one examines the case of a single-phase circuit formed by two conductors of the same geometrical properties as those of an NYM $3 \times 2.5 \mathrm{~mm}^{2}$ cable, with a distance of $1 \mathrm{~m}$ between them

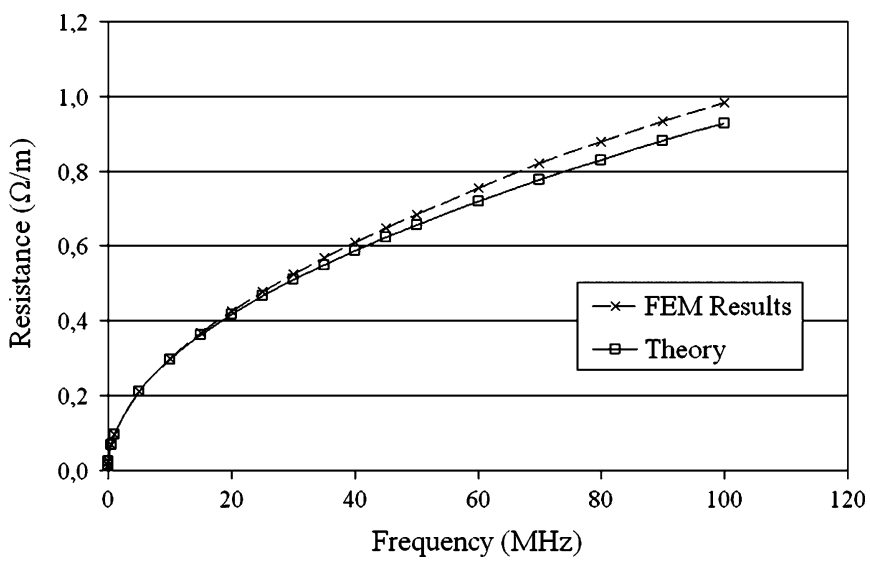

Fig. 6. Circuit resistance per unit length for two conductors with the same geometrical properties as the conductors of an NYM $3 \times 2.5 \mathrm{~mm}^{2}$ cable, at a distance of $1 \mathrm{~m}$ between them. The third conductor is disregarded.

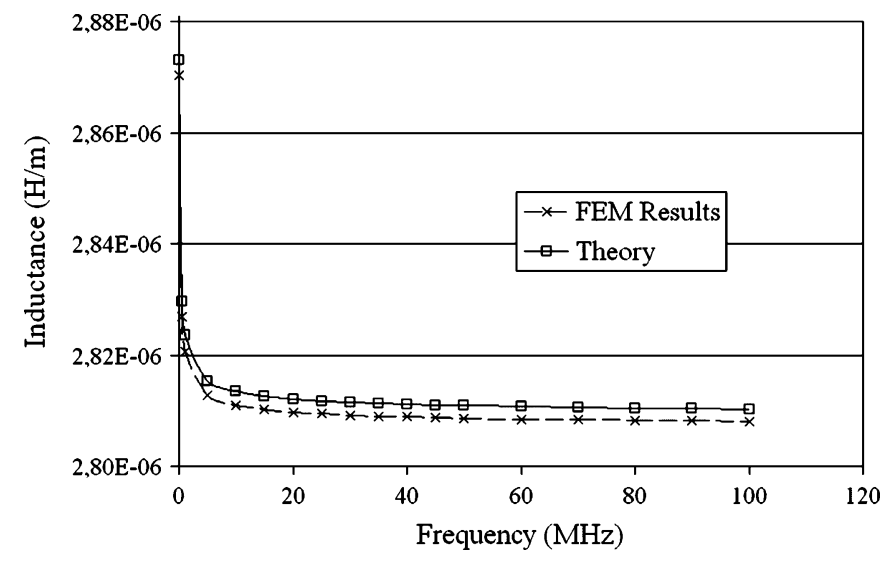

Fig. 7. Circuit inductance per unit length for two conductors with the same geometrical properties as the conductors of an NYM $3 \times 2.5 \mathrm{~mm}^{2}$ cable, at a distance of $1 \mathrm{~m}$ between them. The third conductor is disregarded.

(i.e., a distance long enough for the proximity effect not to contribute to their characteristics). The circuit resistance and inductance per unit length in this case can be seen in Figs. 6 and 7, respectively.

It can be observed in Fig. 6 that FEM results are very close to those theoretically expected, when the proximity effect can be neglected. The remaining deviation (which reaches up to $5.78 \%$ at $90 \mathrm{MHz}$ ) is caused by the imprecision of the theoretical analysis at high frequencies.

In Fig. 7, the almost absolute convergence of theoretical results with those obtained by the finite-element approach is observed once more, regarding the circuit inductance per unit length. In this case, the deviation between theoretical calculations and FEM results remains below $0.20 \%$ for the whole frequency range under study.

In addition to the above, it is also observed in both Figs. 4 and 5 that the presence of the earth does not affect results seriously. Concerning the circuit's resistance, the deviation due to the presence of the earth remains less than $1 \%$ for the whole frequency range under study. Concerning the circuit's inductance, the deviation due to the presence of the earth remains at all frequencies below $0.1 \%$. 


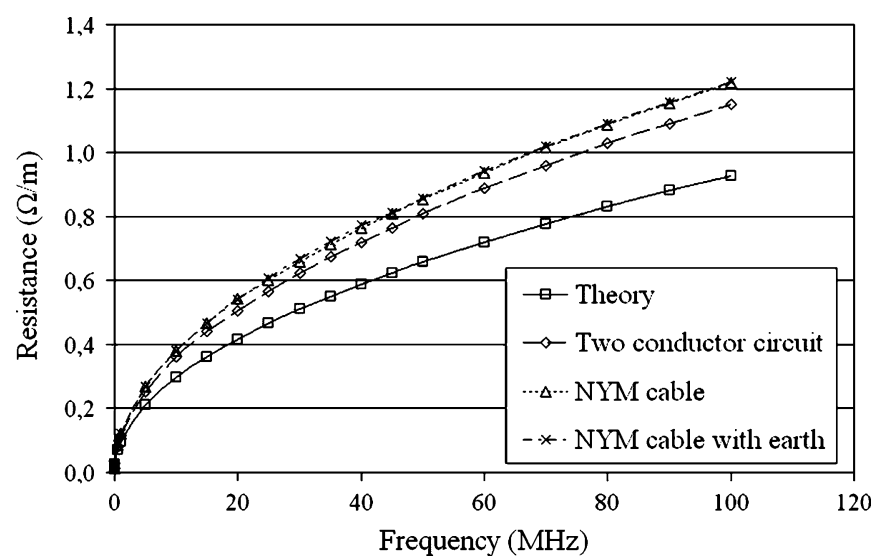

Fig. 8. Circuit resistance per unit length for an NYM $3 \times 2.5 \mathrm{~mm}^{2}$ cable.

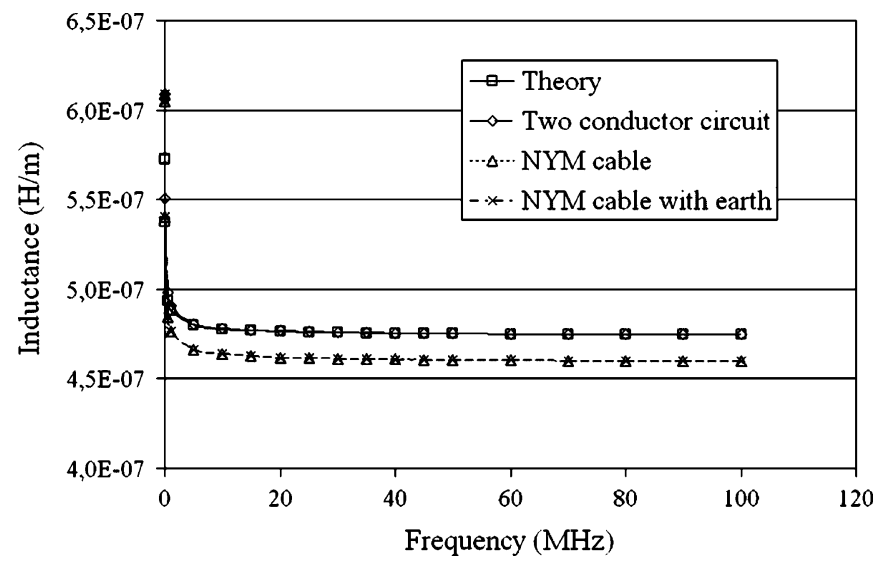

Fig. 9. Circuit inductance per unit length for an NYM $3 \times 2.5 \mathrm{~mm}^{2}$ cable.

This, however, was also expected, due to the circuit's small dimensions compared to its distance from the ground. In a former work by the authors [14], it is shown that the operational series impedances per unit length in the case of a cable of such small dimensions are practically independent from both the value of earth resistivity and cable distance from ground.

\section{Presence of the Third Conductor}

Next, the case of a single-phase circuit formed by two conductors of an NYM $3 \times 2.5 \mathrm{~mm}^{2}$ cable will be considered, whereas the cable's third conductor is grounded. The FEM results for this case are shown in Figs. 8 and 9, regarding, respectively, the cable's resistance and inductance per unit length versus current frequency. Both figures consist of four curves each.

The first one shows the same theoretical results shown in Figs. 4 and 5, respectively, represent the theoretical analysis considering a single-phase circuit and disregarding the presence of the third conductor. The second curve represents the FEM results for the aforementioned case of a single-phase circuit, disregarding again the presence of the third conductor. The two remaining curves represent the FEM results for two different configurations, a configuration of a fully operational NYM $3 \times$ $2.5 \mathrm{~mm}^{2}$ cable disregarding the presence of the earth, and an-

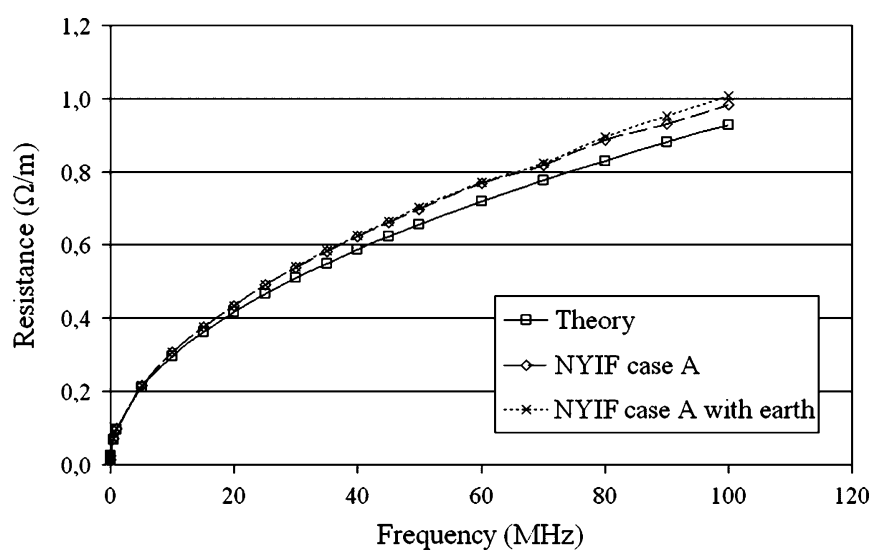

Fig. 10. Circuit resistance per unit length for an NYIF $3 \times 2.5 \mathrm{~mm}^{2}$ cable (case A).

other one of the same kind, whereas the cable lies $0.5 \mathrm{~m}$ above ground (with an earth resistivity of $100 \Omega \cdot \mathrm{m}$ ).

In Fig. 8, it is observed as expected that the cable resistance is greater than that of a single-phase circuit disregarding the presence of the third conductor. Once again, this occurs due to the proximity effect caused by the third conductor, being very close to the other two.

Concerning the cable inductance, a slight reduction in its value is observed in Fig. 9, compared to that of a single-phase circuit disregarding the third conductor. This, however, is also to be expected as the third conductor of the cable is grounded and serves thus as a shield reducing the mutual inductance among the two conductors forming the circuit.

Moreover, in both Figs. 8 and 9, it can be seen once again that the presence of the earth only has a negligible effect on the cable's series impedances, as the distance between the cable's conductors is very small compared to its distance from the ground.

\section{E. NYIF Cable Geometry}

In Figs. 10-13, the case of an NYIF $3 \times 2.5 \mathrm{~mm}^{2}$ cable is considered, used in the same configuration as the NYM cable of Section IV-D, namely with two of its conductors forming a single-phase circuit, whereas the third conductor is grounded. In the case of this cable type, the axial symmetry of the NYM-conductors' geometry does not exist; therefore, we examine two different configurations. In the first one (named "case A" in the respective figures), the single-phase circuit is formed by conductors 1 and 2 of Fig. 1, whereas in the second one (named "case B" in the respective figures), the single-phase circuit is formed by conductors 1 and 3, respectively.

Figs. 10 and 12 depict FEM results considering the cable's resistance per unit length for both cases, whereas Figs. 11 and 13 show the respective cable's inductance per unit length for both cases. For every case, there are also theoretical results, as well as results considering the presence of the earth, whereas the cable is located $0.5 \mathrm{~m}$ above ground and the earth resistivity is $100 \Omega \cdot \mathrm{m}$. The theoretical results refer in any case to the electrical parameters of the respective single-phase circuit, disregarding the presence of the cable's third conductor. 


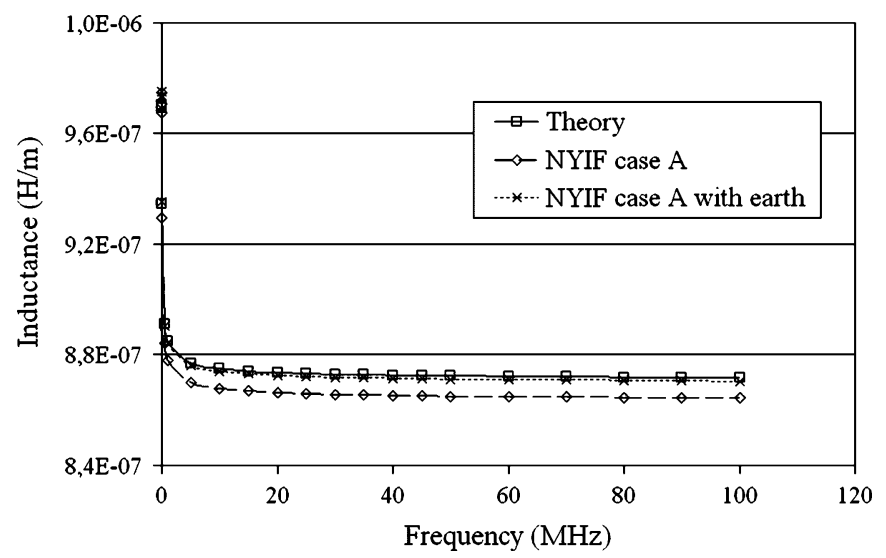

Fig. 11. Circuit inductance per unit length for an NYIF $3 \times 2.5 \mathrm{~mm}^{2}$ cable (case A).

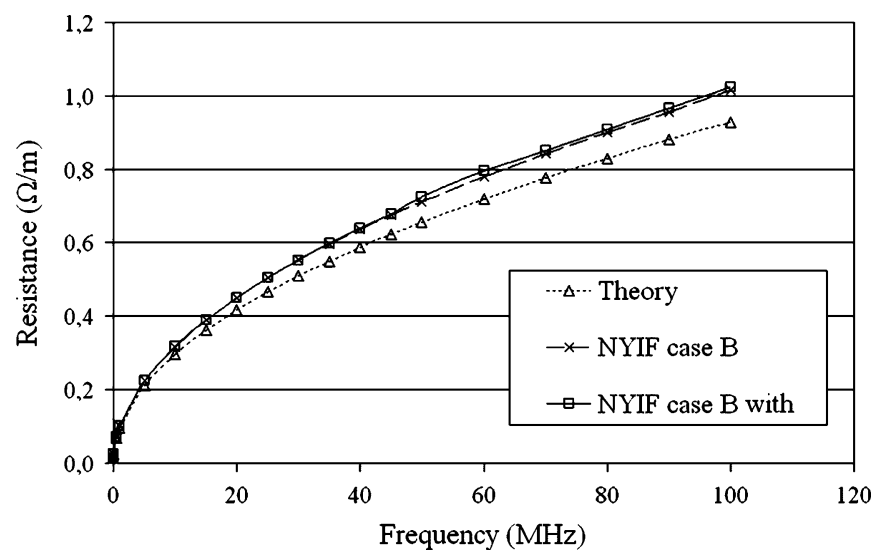

Fig. 12. Circuit resistance per unit length for an NYIF $3 \times 2.5 \mathrm{~mm}^{2}$ cable (case B).

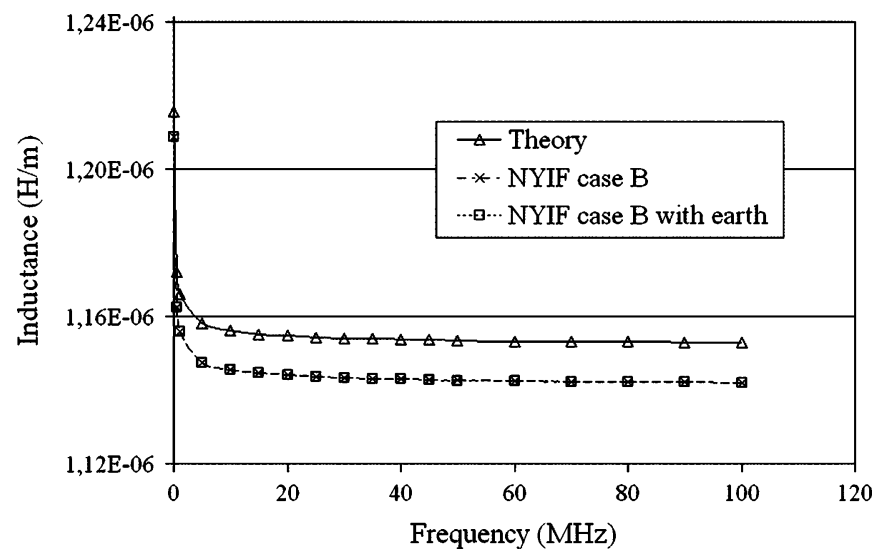

Fig. 13. Circuit inductance per unit length for an NYIF $3 \times 2.5 \mathrm{~mm}^{2}$ cable (case B).

As can be seen in Figs. 10 and 12, the theoretical results disregarding the cable's third conductor are for this cable type closer to FEM results, than in the case of an NYM cable. More specifically, the deviation for the case $\mathrm{A}$ is at all frequencies less than $6.5 \%$, whereas the respective deviation for case B is at all frequencies less than $8.7 \%$. This occurs because the distance between the conductors of an NYIF cable is more than twice the respective distance between the conductors of an NYM cable; therefore, the proximity effect is weaker in the case of the NYIF cable.

Moreover, differences due to the configuration of the cable are very small concerning the cable's resistance per unit length (the maximum deviation is $3.66 \%$ at $20 \mathrm{MHz}$ ). This occurs because the alteration of the cable configuration affects its resistance only through the proximity effect, whose contribution is generally small in the overall value of the resistance.

On the contrary, Figs. 11 and 13 show that the alteration of the cable's configuration strongly affects the value of its inductance (with a constant deviation of about $24 \%$ among the two different configurations). This, however, is also to be expected, as the inductance of a cable depends heavily on the distance between the conductors.

Finally, in all figures, we observe once more that the operational electrical parameters of cables of such small dimensions are practically independent from the presence of the earth.

\section{CONCLUSION}

A thorough theoretical analysis is presented in this work concerning the calculation of the lumped electrical parameters of LV power distribution cables. The analysis is based on existing literature, and the frequency dependency of the parameters is taken into account whenever possible. In addition, a finite-element approach is used to extract the series impedances per unit length of the same cables, taking into account the geometric configuration, skin and proximity effect, as well as eddy currents flowing in other possibly existing conducting paths. The series impedances per unit length are calculated for a frequency range up to $100 \mathrm{MHz}$, in order to cover for the typical spectrum used in today's power-line communication applications.

The FEM results are, at first, theoretically validated for simple conductor geometries, whereas, consequently, the finite-element approach is used to derive the electrical parameters per unit length of conductor geometries which correspond to the operational conditions of the aforementioned cables. The inability of the existing theory to account for all phenomena in the frequency range under study leads to expected differences in the FEM results compared to theoretical calculations, which are denoted and explained.

\section{REFERENCES}

[1] D. Anastasiadou and T. Antonakopoulos, "An experimental setup for characterizing the residential power grid variable behavior," presented at the 6th Int. Symp. Power-Line Communications and Its Applications, Athens, Greece.

[2] I. C. Papaleonidopoulos, C. G. Karagiannopoulos, N. J. Theodorou, C. E. Anagnostopoulos, and I. E. Anagnostopoulos, "Modelling of indoor low voltage power-line cables in the high frequency range," presented at the 6th Int. Symp. Power-Line Communications and Its Applications, Athens, Greece.

[3] M. Zimmermann and K. Dostert, "A multipath model for the powerline channel,” IEEE Trans. Commun., vol. 50, no. 4, pp. 553-559, Apr. 2002.

[4] L. Heinhold, Power Cables and Their Application-Part 1. Berlin, Germany: Siemens Aktiengesellschaft, 1993, p. 11, p. 331, p. 333.

[5] R. E. Matick, Transmission Lines for Digital and Communication Networks. Piscataway, NJ: IEEE Press, 1995, pp. 93-106, 317, 344-350.

[6] O. I. Elgerd, Electric Energy Systems Theory: An Introduction. New Delhi, India: Tata/McGraw-Hill, 1978, pp. 158-161.

[7] R. Arora and W. Mosch, High Voltage Insulation Enginnering. New Delhi, India: New Age International, 2004, p. 242. 
[8] A. von Hippel, Dielectric Materials and Applications. Boston, MA: Artech House, 1994, pp. 329-330.

[9] C. R. Paul, Analysis of Multiconductor Transmission Lines. New York: Wiley, 1994, p. 159.

[10] M. S. Naidu and V. Kamaraju, High Voltage Engineering, 2nd ed. New York: McGraw-Hill, 1995, p. 85.

[11] S. Tsuzuki, S. Yamamoto, T. Takamatsu, and Y. Yamada, "Measurement of Japanese indoor power-line channel," in Proc. 5th Int. Symp. Power-Line Communications and Its Applications, Malmö, Sweden, Apr. 4-6, 2001, pp. 79-84.

[12] D. Labridis and P. Dokopoulos, "Finite element computation of field, losses and forces in a three-phase gas cable with non-symmetrical conductor arrangement," IEEE Trans. Power Del., vol. 3, no. 4, pp. 1326-1333, Oct. 1988.

[13] D. G. Triantafyllidis, G. K. Papagiannis, and D. P. Labridis, "Calculation of overhead transmission line impedances: a finite element approach," IEEE Trans. Power Del., vol. 14, no. 1, pp. 287-293, Jan. 1999.

[14] G. T. Andreou and D. P. Labridis, "Simulation of a LV power distribution cable as communication medium-a finite element approach," in Proc. IEEE St. Petersburg PowerTech, St. Petersburg, Russia, 2005.

[15] D. P. Labridis, "Comparative presentation of criteria used for adaptive finite element mesh generation in multiconductor eddy current problems," IEEE Trans. Magn., vol. 36, no. 1, pp. 267-280, Jan. 2000.
Georgios T. Andreou (S'98-A'02-M'04) was born in Thessaloniki, Greece, on August 16, 1976. He received the Dipl.-Eng. degree from the Department of Electrical and Computer Engineering, Aristotle University of Thessaloniki, Thessaloniki, in 2000.

Since 2001, he has been a Postgraduate Student at the Department of Electrical and Computer Engineering, Aristotle University of Thessaloniki. His special interests are power system analysis and power-line communications.

Dimitris P. Labridis (S'88-M'90-SM'00) was born in Thessaloniki, Greece, on July 26, 1958. He received the Dipl.-Eng. and the Ph.D. degrees from the Department of Electrical Engineering at the Aristotle University of Thessaloniki, Thessaloniki, in 1981 and 1989, respectively.

During 1982-2001, he was a Research Assistant and then a Lecturer and Assistant Professor at the Department of Electrical Engineering, Aristotle University of Thessaloniki. Since 2001, he has been Associate Professor in the same department. His special interests are power system analysis with special emphasis on the simulation of transmission and distribution systems, electromagnetic and thermal field analysis, numerical methods in engineering, artificial intelligence applications in power systems, and power-line communications. 